\title{
A EXPOSIÇÃO DE TRABALHADORES FLORESTAIS AO CALOR DURANTE O FENÔMENO EL NIÑO GODZILLA NA AMAZÔNIA
}

\author{
THE FOREST WORKERS HEAT EXPOSURE DURING EL NIÑO GODZILLA PHENOMENON IN \\ $A M A Z O N$
}

\author{
Kauê Augusto Oliveira Nascimento', Niro Higuchi' ${ }^{2}$, Fabiano Emmert ${ }^{3}$ \\ 1 Universidade Federal do Paraná, Curitiba, Paraná, Brasil - kaue_nascimento@hotmail.com \\ ${ }^{2}$ Instituto Nacional de Pesquisas da Amazônia, Manaus, Amazonas, Brasil - higuchi.niro@gmail.com \\ 33 Universidade Federal Rural da Amazônia, Belém, Pará, Brasil -fabianoemmert@yahoo.com.br
}

RESUMO

O conforto térmico laboral está entre 20 e $24^{\circ} \mathrm{C}$, porém na Amazônia as médias diurnas superam os $28^{\circ} \mathrm{C}$, ou seja, há desconforto térmico no trabalho. Para acentuar, a ocorrência de fenômenos extremos El Niño relacionados à mudança climática, vêm causando aumento das temperaturas na região e os efeitos recairão sobre a saúde e capacidade dos trabalhadores. O potencial desenvolvimento do manejo florestal e o bem-estar laboral vão de encontro a previsões de fenômenos extremos na Amazônia, o que justifica a realização deste trabalho. Este estudo teve como objetivo a coleta de variáveis ambientais durante o El Niño Godzilla (2015-2016) e comparação com outros períodos. $\mathrm{O}$ estudo foi conduzido no município de Itapiranga, Amazonas, Brasil, coordenadas latitude $02^{\circ} 75^{\prime}$ $\mathrm{S}$ e longitude $58^{\circ} 47^{\prime} \mathrm{W}$ durante novembro de 2015. As variáveis coletadas foram: IBUTG (Índice de bulbo úmido e termômetro de globo) $\left({ }^{\circ} \mathrm{C}\right)$; temperatura do $\operatorname{ar}\left({ }^{\circ} \mathrm{C}\right)$, temperatura de globo $\left({ }^{\circ} \mathrm{C}\right)$ e umidade relativa do ar $(\%)$. As análises utilizaram testes $\mathrm{t}$ de Student para dados pareados e não-pareados, interpretados pela tabela de probabilidade do IPCC (2010). Os resultados demonstraram diferenças significativas para todas as variáveis e cenários: temperaturas maiores e umidade menor durante o El Niño Godzilla. As adequações práticas da exposição ao calor acarretam em maior bem-estar dos trabalhadores, com consequentes melhorias à saúde, à segurança e ao desempenho das operações, sob o cenário atual e de mudança climática.

PALAVRAS-CHAVE: Manejo florestal, Mudança climática, Saúde do trabalho.

\section{ABSTRACT}

The thermal comfort of work is between 20 and $24^{\circ} \mathrm{C}$, but in the Amazon the daytime averages exceeds $28^{\circ} \mathrm{C}$, so there is thermal discomfort at work. Furthermore, the occurrence of extreme El Niño phenomena related to climate change have been causing an increase in temperatures in the region and the effects will affect the health and capacity of workers. The potential development of forest management and labor well-being disagrees with predictions of extreme phenomena in the Amazon, which justifies the accomplishment of this work. This study aimed to collect environmental variables during the El Niño Godzilla (2015-2016) and do comparisons with other periods. The study was conducted in the municipality of Itapiranga, Amazonas, Brazil, coordinates latitude $02^{\circ} 75^{\prime} \mathrm{S}$ and longitude $58^{\circ} 47^{\prime} \mathrm{W}$ during November 2015. The variables collected were: WBGT (Wet bulb globe temperature), air temperature $\left({ }^{\circ} \mathrm{C}\right)$, globe temperature $\left({ }^{\circ} \mathrm{C}\right)$ and relative air humidity $(\%)$. The analysis used Student's t-tests for paired and unpaired data, interpreted by the IPCC (2010) probability table. The results showed significant differences for all variables and scenarios: higher temperatures and lower humidity during El Niño Godzilla. The practical adequacy of heat exposure results in greater worker well-being, with consequent improvements in health, safety, and operational performance, under the current and climate change scenarios.

KEY WORDS: Forest management, Climate change, Occupational health. 


\section{INTRODUÇÃO}

A temperatura média global tem aumentado e é estimado aumento entre 1,8 e $4^{\circ} \mathrm{C}$ até o ano de 2100 , segundo o Intergovernmental Panel on Climate Change (IPCC, 2007). Até meados dos anos 2000, as pesquisas sobre mudança climática eram focadas em impactos ambientais e ecossistêmicos (KJELLSTROM, 2009). O trabalho de McMichael et al. (1996) foi pioneiro no estudo dos efeitos da mudança climática à saúde humana, após isso o IPCC passou a incorporar o tema em seus relatórios (KJELLSTROM, 2009). Eventos climáticos (ondas de calor e secas extremas) como o El Niño são responsáveis por diversas mortes e doenças nas populações afetadas (HUBLER et al., 2008; KJELLSTROM \& CROWE, 2011).

O fenômeno El Niño conceitua-se como o aquecimento atípico da superfície do Oceano Pacífico ao longo das baixas latitudes, este fenômeno gera o aumento da evaporação da superfície do oceano e com isso a pressão atmosférica aumenta, gerando o componente atmosférico conhecido por Oscilação Sul, por essa razão, o fenômeno é conhecido por ENSO (El NiñoSouthern Oscillation) (TRENBERTH, 1997). O El Niño gera uma queda da precipitação durante a estação chuvosa na Amazônia (JIMÉNEZ-MUÑOZ et al., 2013). Eventos climáticos de seca extrema, como o El Niño Modoki de 2010 causaram um aumento de temperatura da superfície terrestre por um período prolongado na região (JIMÉNEZMUÑOZ et al., 2013). Visivelmente estes fenômenos têm se tornado mais frequentes, agressivos e duradouros nos últimos anos, com destaque para as secas de 2005 e 2010 na região amazônica (JIMÉNEZ-MUÑOZ et al., 2013).

Dentre os possíveis riscos à saúde, o estresse térmico e seus relativos tem posição de destaque (KJELLSTROM et al., 2009a). No decorrer dos anos a mudança climática tende a gerar maior exposição ao calor para bilhões de pessoas em países tropicais e muito provavelmente irá afetar as pessoas mais pobres que trabalham em atividades expostas a estas condições, como agricultura, operações florestais e construção civil, causando a degeneração da saúde destes trabalhadores (KJELLSTROM, 2009).

O trabalho florestal é considerado um dos mais desgastantes e perigosos no mundo atual. Esta atividade demanda atenção e preparo físico dos trabalhadores, especialmente na exploração seletiva de madeira. Nestas circunstâncias o trabalho é semi-mecanizado, exige grande contingente e carga física aos trabalhadores em uma floresta com denso sub-bosque e em condições térmicas desfavoráveis (EMMERT, 2014). O conforto térmico no trabalho em regiões tropicais está entre 20 e $24^{\circ} \mathrm{C}$ segundo IIDA (1990), porém na Amazônia Central as médias diurnas superam os $28^{\circ} \mathrm{C}$ (INMET, 2016). Portanto, há no mínimo desconforto térmico para o trabalho. Para agravar a situação, a ocorrência de fenômenos El Niño extremos vem causando aumento das temperaturas, de acordo com Jiménez-Muñoz et al. (2013), e parte dos efeitos recairão sobre a saúde e a capacidade laboral dos trabalhadores (KJELLSTROM et al., 2009a). Ainda que, os efeitos ainda sejam pouco conhecidos, necessita-se de estudos para que ações adequações ergonômicas sejam tomadas antecipadamente (IIDA, 1990). Em seu estudo, Kjellstrom et al. (2009a) concluíram que mais horas de trabalho ou mais trabalhadores serão necessários para compensar as perdas econômicas das doenças ocupacionais, ações de prevenção e queda de desempenho dos trabalhadores devido à mudança climática.

O potencial desenvolvimento do manejo florestal na região e o bem-estar laboral na exploração de madeira vão de encontro a previsões de fenômenos extremos e aumento das temperaturas médias na Amazônia, desafios trazidos pela mudança climática, com a tendência de danos à saúde dos trabalhadores pela exposição ao calor, panorama este que justifica a realização do estudo. Este estudo teve como objetivo a coleta de variáveis ambientais durante o fenômeno extremo El Niño (20152016) e comparação com dados históricos do clima local e com um estudo realizado na área estudada, em uma época sem ocorrência de fenômenos (2010).

\section{MATERIAL E MÉTODOS}

\section{Área de estudo}

O estudo foi conduzido na unidade de produção anual (UPA) da safra de 2015, contemplando as operações florestais de corte, pré-arraste, arraste e de pátios (intermediário e principal). A UPA localiza-se no município de Itapiranga, nas coordenadas latitude $02^{\circ} 75^{\prime} \mathrm{S}$ e longitude $58^{\circ} 47^{\prime} \mathrm{W}$. O clima na área de estudo é classificado como tropical úmido (Af), com precipitação média anual de $2200 \mathrm{~mm}$ e sem estação seca definida de acordo com a classificação de Koppen-Geiger (ALVARES et al., 2013). A temperatura média é de $28^{\circ} \mathrm{C}$ e umidade relativa do ar de $80 \%$ (INMET, 2016). Segundo Leitão Filho (1987) a vegetação é considerada Floresta de Terra Firme, fitofisionomia predominante na Amazônia. 


\section{Coleta de dados}

Os dados foram coletados entre os dias 04/11/2015 e $02 / 12 / 2015$, com algumas intermitências devido às folgas na escala de trabalho. A maioria das variáveis foram coletadas simultaneamente e classificadas por hora ao longo do turno de trabalho. Foram coletadas variáveis ambientais e o índice de bulbo úmido e termômetro de globo (IBUTG), para avaliação ocupacional da exposição ao calor. A fórmula do índice IBUTG constitui-se de três variáveis para ambiente externo e com carga solar.

Ambiente externo com carga solar:

$$
\text { IBUTG = 0,7 Tbu+0,2 Tg+0,1 Tbs }
$$

Em que: $\mathrm{Tbu}=$ temperatura de bulbo úmido $\left({ }^{\circ} \mathrm{C}\right) ; \mathrm{Tg}=$ temperatura de globo $\left({ }^{\circ} \mathrm{C}\right)$; e Tbs = temperatura de bulbo $\operatorname{seco}\left({ }^{\circ} \mathrm{C}\right)$.

Para mensurar IBUTG foi utilizado um equipamento digital modelo HT30 portátil, da fabricante Extech Instruments. Seguindo os procedimentos de coleta para trabalho contínuos e ao ar livre, as medidas do índice foram tomadas a cada hora. Em campo, o equipamento foi colocado em posição vertical, na altura média da cabeça de um trabalhador florestal (cerca de 1,70 m). 0 equipamento foi posicionado o mais próximo possível do trabalhador sob avaliação, levando em conta a segurança e evitando interferências nas operações. Após o tempo de estabilização, foi coletado o índice IBUTG conforme o procedimento a seguir:

1. O IBUTG foi coletado no começo de cada hora de trabalho, junto a outras variáveis ambientais mensuradas pelo instrumento: umidade relativa do ar (\%), temperatura do $\operatorname{ar}\left({ }^{\circ} \mathrm{C}\right)$ e temperatura de globo $\left({ }^{\circ} \mathrm{C}\right)$; e

2. Adicionalmente foram coletadas temperaturas em IBUTG ao final de cada ciclo operacional analisado.

As variáveis coletadas foram: temperatura do $\operatorname{ar}\left({ }^{\circ} \mathrm{C}\right)$, temperatura de globo $\left({ }^{\circ} \mathrm{C}\right)$ e umidade relativa do ar (\%). Os dados coletados foram comparados com valores médios de uma série histórica de 30 anos, entre 19852014 para o mês de novembro, de forma a verificar os efeitos ambientais do fenômeno El Niño Godzilla (20152016), presente durante a coleta de dados. O banco de dados do Instituto Nacional de Meteorologia (INMET) foi consultado e utilizado neste estudo, tendo como referência a estação meteorológica do município de Itacoatiara, a mais próxima da área de estudo.
Foram comparadas também variáveis ambientais coletadas em 2010 por Emmert (2014) com as do presente estudo (2015). Estes dados foram coletados no mesmo mês (novembro), com equipamento idêntico, em uma área próxima e pertencente à mesma empresa. Sabe-se que, ao final do ano de 2010 os eventos climáticos que estavam ocorrendo já haviam dissipado e o tempo na região havia normalizado (MARENGO et al., 2011). Portanto, as análises feitas por Emmert (2014) ocorreram em condição típica de tempo.

Por fim, foram estimados os efeitos do fenômeno $E$ I Niño Godzilla no regime de pausas estipulado pela Norma Regulamentadora no 15 (NR-15), que determina os limites de exposição ao calor no trabalho (BRASIL, 1978). Esta última análise foi feita a exemplo do trabalho realizado por Kjellstrom et al. (2009a), em que foram contabilizadas as pausas para descanso exigidas para cada cenário e avaliadas as diferenças de produtividade, em função do regime de pausas exigido pela normativa vigente.

\section{Análise de dados}

Os dados deste trabalho foram analisados por meio do teste $\mathrm{t}$ de Student para dados pareados e não-pareados, com uso do software Past.exe. Os níveis de significância das probabilidades foram interpretados segundo a tabela desenvolvida pelo Painel Intergovernamental de Mudanças Climáticas, IPCC (2010), descritos na Tabela 1.

Tabela 1. Escalas de probabilidade (p) para as diferenças estatísticas significativas deste estudo

\begin{tabular}{cc}
\hline \multicolumn{2}{c}{ Escalas de probabilidade - Diferença significativa } \\
\hline Intervalos de $p$ & Interpretação \\
$0,01-0,000$ & Praticamente certa \\
$0,1-0,000$ & Muito provável \\
$0,34-0,000$ & Provável \\
$0,66-0,34$ & Tão provável quanto improvável \\
$1-0,67$ & Improvável \\
$1-0,9$ & Muito improvável \\
$1-0,99$ & Excepcionalmente improvável \\
\hline
\end{tabular}

Fonte: Adaptada de IPCC (2010).

\section{RESULTADOS E DISCUSSÃO}

\section{El Niño Godzilla versus Série Histórica (1985-2014)}

Foi consultado o banco de dados do Instituto Nacional de Meteorologia (INMET) e utilizados dados de variáveis meteorológicas da estação do município de Itacoatiara, Amazonas, para uma série histórica de 30 anos (1985- 
2014). Os dados utilizados, foram referentes aos mesmos dias do ano em que houve coleta de dados para a realização deste trabalho, totalizando 21 dias. Estes dados foram comparados com as variáveis coletadas em campo e aplicado o teste $\mathrm{t}$ para dados pareados, conforme a Tabela 2.

Tabela 2. Comparação entre variáveis meteorológicas de uma série histórica com as coletadas em campo

\begin{tabular}{cccc}
\hline Variáveis & Média & t & p-valor \\
\hline T. $\operatorname{ar}\left({ }^{\circ} \mathrm{C}\right) 30$ anos & 27,80 & 7,16 & 0,0000 \\
\hline T. $\operatorname{ar}\left({ }^{\circ} \mathrm{C}\right)$ El Niño & 31,29 & & \\
\hline UR(\%) 30 anos & 81,01 & \multirow{2}{*}{0,91} & 0,0001 \\
UR(\%) El Niño & 67,72 & & \\
\hline T. máx $\left({ }^{\circ} \mathrm{C}\right) 30$ anos & 33,27 & \multirow{2}{*}{1,75} & 0,0940 \\
\hline T. máx $\left({ }^{\circ} \mathrm{C}\right)$ El Niño & 34,58 & & \\
\hline T. mín. $\left({ }^{\circ} \mathrm{C}\right) 30$ anos & 23,87 & \multirow{2}{*}{5,64} & 0,0000 \\
\hline T. mín. $\left({ }^{\circ} \mathrm{C}\right)$ El Niño & 26,08 & & \\
\hline
\end{tabular}

Com base nos resultados, pode-se afirmar que houve diferenças altamente significativas entre as médias das variáveis da série histórica com as coletadas em campo, sendo praticamente certas as diferenças em temperatura do ar, umidade relativa do ar e temperatura mínima. Para a variável temperatura máxima, os resultados demonstraram que a diferença é muito provável.

As temperaturas médias durante o fenômeno foram maiores que as médias históricas, enquanto que a umidade relativa do ar resultou em valores menores que as médias históricas. Isso demonstrou os efeitos ambientais drásticos causados pelo fenômeno El Niño Godzilla na Amazônia, de mesma natureza e de efeitos similares ao El Niño Modoki ocorrido em 2010, baseado nos resultados de Jiménez-Muñoz et al. (2013). Deve-se enfatizar que historicamente os meses de agosto e setembro são os mais secos e outubro é o mais quente (INPE, 2016; INMET, 2016), ou seja, durante estes meses de clima mais extremo os efeitos do El Niño provavelmente foram mais prejudiciais ao bem-estar laboral.

\section{El Niño Godzilla versus período sem fenômeno (novembro de 2010)}

Foi realizada uma análise preliminar entre os dados históricos para a região e os dados coletados por Emmert (2014), para validar a tipicidade das condições ambientais em novembro de 2010. Foram avaliadas as variáveis temperatura do ar e umidade relativa do ar, pois foram as únicas variáveis coincidentes entre o banco de dados do INMET e os dados de Emmert (2014). Foi realizado Teste $t$ para dados pareados, pois os dados referiram-se aos mesmos dias do ano (Tabela 3 ).

Os dados de temperatura do ar coletados em 2010 apresentaram médias muito similares, com uma probabilidade pouco provável de diferenças estatísticas entre os dados ( $p$-valor: 0,5468). Os dados de umidade relativa do ar resultaram em diferença muito provável ( $p$ valor: 0,0781). A UR (\%) no ano de 2010, foi em média $6 \%$ superior à média histórica. Com isso, pode-se supor que novembro de 2010 foi um período mais chuvoso que o usual para este mês. Apesar da temperatura apresentar resultados menores que em 2015, a maior frequência de chuvas e o consequente aumento de umidade do ar, provavelmente afetaram o andamento das operações e o desempenho dos trabalhadores florestais.

Tabela 3. Comparação entre variáveis ambientais de uma série histórica (1985-2014) com os dados coletados por Emmert (2014), em 2010

\begin{tabular}{cccc}
\hline Variáveis & Média & $\mathbf{t}$ & p-valor \\
\hline Temp. $\operatorname{ar}\left({ }^{\circ} \mathrm{C}\right) 30$ anos & 27,81 & 0,60 & 0,5468 \\
Temp. $\operatorname{ar}\left({ }^{\circ} \mathrm{C}\right) 2010$ & 28,05 & & \\
\hline UR $(\%) 30$ anos & 81,12 & \multirow{2}{*}{2,46} & 0,0781 \\
UR (\%) 2010 & 87,24 & & \\
\hline
\end{tabular}

Em 2010, também foram coletados por Emmert (2014) dados em IBUTG e de outras variáveis ambientais. Dessa forma, foi possível verificar as diferenças ambientais entre o período de El Niño Godzilla com o ano de 2010, sem efeitos de fenômenos naturais. Foi aplicado o teste t para dados não-pareados e os resultados apresentados na Tabela 4.

Tabela 4. Comparação entre as variáveis ambientais do ano de 2010 com as coletadas durante o El Niño Godzilla, na área de estudo

\begin{tabular}{cccc}
\hline Variáveis & Média & t & p-valor \\
\hline IBUTG $\left({ }^{\circ} \mathrm{C}\right) 2010$ & 26,87 & \multirow{2}{*}{6,33} & 0,0000 \\
IBUTG $\left({ }^{\circ} \mathrm{C}\right)$ El Niño & 28,05 & & \\
\hline Temp. $\operatorname{ar}\left({ }^{\circ} \mathrm{C}\right) 2010$ & 28,38 & \multirow{2}{*}{8,60} & 0,0000 \\
Temp. $\operatorname{ar}\left({ }^{\circ} \mathrm{C}\right)$ El Niño & 31,27 & & \\
\hline Temp. globo $\left({ }^{\circ} \mathrm{C}\right) 2010$ & 29,76 & \multirow{2}{*}{7,15} & 0,0000 \\
Temp. globo $\left({ }^{\circ} \mathrm{C}\right)$ El Niño & 34,29 & & \\
\hline UR $(\%) 2010$ & 81,09 & \multirow{2}{*}{$-7,65$} & 0,0000 \\
\hline UR $(\%)$ El Niño & 67,82 & & \\
\hline
\end{tabular}


Os resultados apontaram que houve diferenças significativas entre os dados de Emmert (2014) com os do presente estudo, destacando-se as médias de temperatura do ar, temperatura de globo e umidade relativa do ar. Além disso, encontrou-se diferença média superior a $1^{\circ} \mathrm{C}$ em IBUTG.
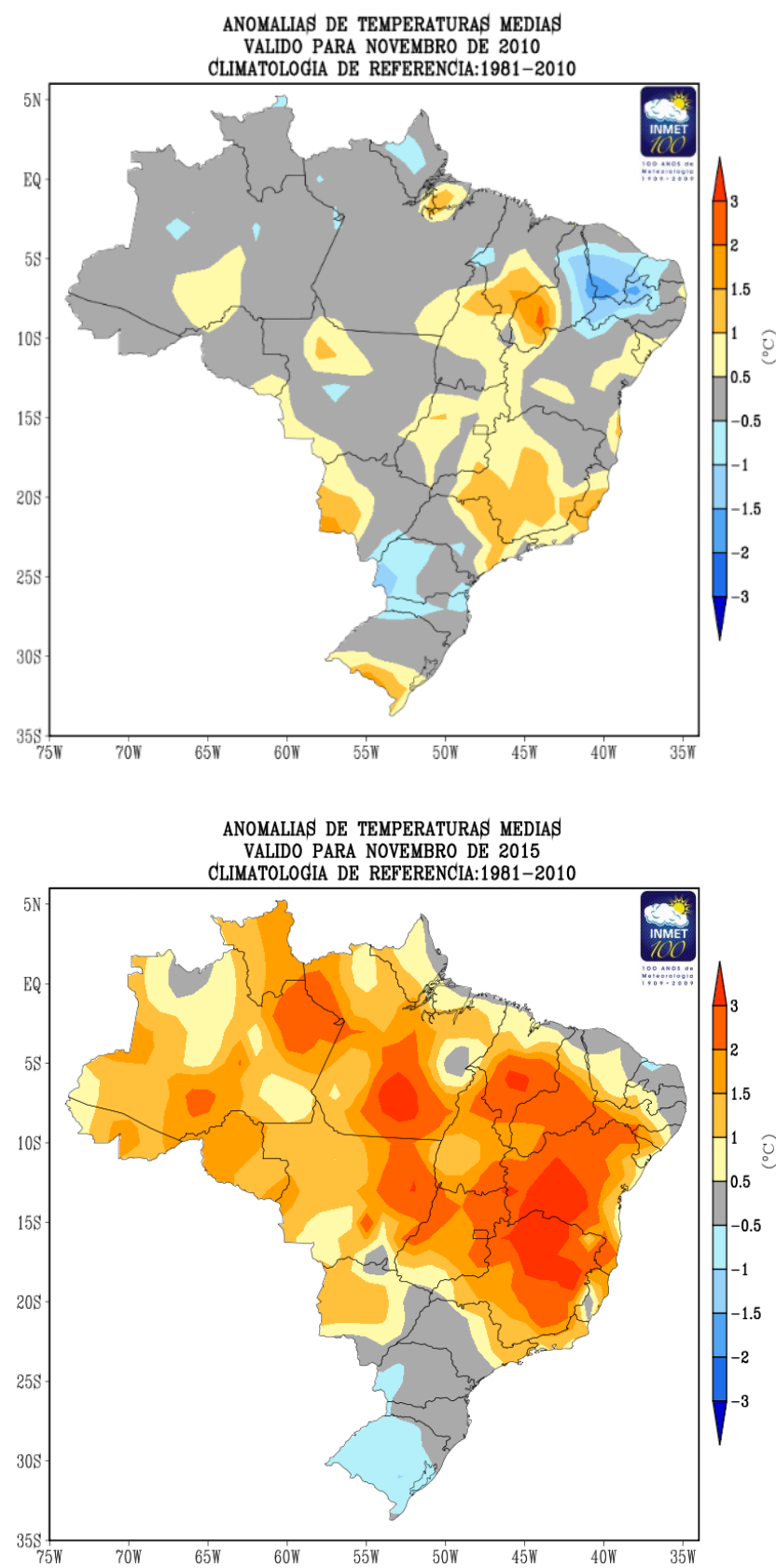

Figura 1. Diferenças entre as anomalias de temperatura para o mês de novembro, nos anos de 2010 e 2015 (esquerda para direita).

Fonte: INMET (2016).

Estes resultados demonstraram que as diferenças entre as médias para todas as variáveis foram praticamente certas, sendo que as variáveis temperatura do ar e temperatura de globo apresentaram médias significativamente acima dos valores presentes em novembro de 2010, enquanto a umidade relativa do ar foi significativamente menor que em 2010. As implicações possíveis destes resultados são diversas, uma vez que estas condições ambientais atípicas afetam o desempenho e a saúde de trabalhadores que realizaram árduas atividades ao ar livre, além da falta de adaptação fisiológica a condições extremas, citadas por Kjellstrom et al. (2009a) e Kjellstrom e Crowe (2011).

Para visualização das diferenças entre as temperaturas de novembro de 2010 e novembro de 2015, foram representadas duas imagens de anomalias de calor com base na média histórica para todo o país (Figura 1).

A partir da Figura 1 percebeu-se que as anomalias de temperatura foram superiores em novembro de 2015 em relação a 2010, apresentando entre 2 e $3^{\circ} \mathrm{C}$ de anomalia positiva na região da área estudada e em diversos locais, abrangendo praticamente toda a porção tropical do Brasil. É notável que em 2010 as anomalias positivas apresentaram distribuição e intensidade reduzidas, resultando em ausência de anomalias de temperatura na área de estudo. Em novembro de 2010, o El Niño modoki que terminou naquele ano já havia se dissipado (MARENGO et al., 2011), enquanto que em 2015 o El Niño Godzilla estava ativo durante a coleta de dados.

Desse modo, estes resultados demonstraram os efeitos ambientais significativos destes fenômenos extremos na Amazônia, corroborados por Marengo et al. (2011) e Jiménez-Muñoz et al. (2013). A Figura 2 ilustrou o comportamento das temperaturas ao longo da jornada de trabalho, na área de manejo florestal, para os dois períodos.

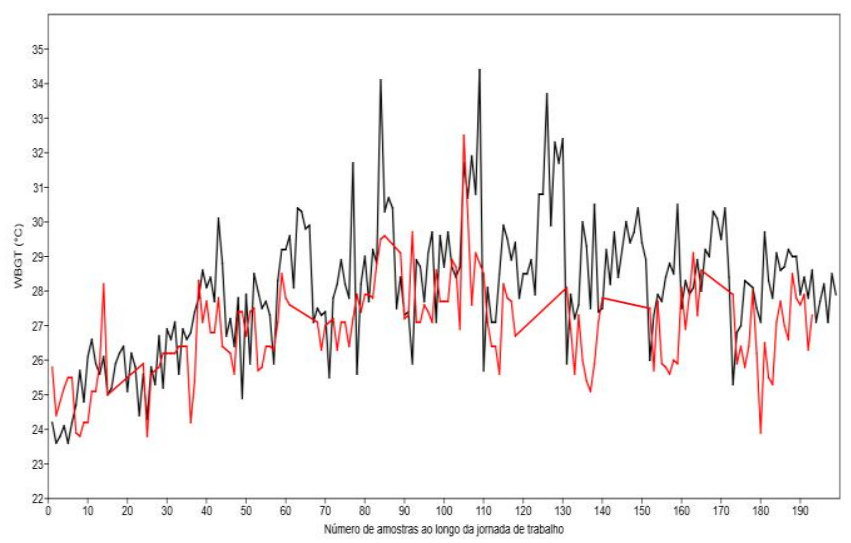

Figura 2. Temperaturas em IBUTG coletadas no ano de 2010 (vermelha) e durante o El Niño Godzilla em 2015 (preta), na área de manejo florestal.

Pode-se afirmar a partir do gráfico que o 
comportamento das temperaturas em IBUTG ao longo da jornada de trabalho, foi similar para os dois períodos. Entretanto, os valores de temperatura foram visivelmente diferentes, com a presença de picos de temperatura durante os horários mais quentes (10-15 h) para o El Niño. A diferença média entre os valores em IBUTG para os dois períodos foi de $1,18^{\circ} \mathrm{C}$, o que indicou possíveis diferenças na classificação do regime de pausas da NR-15 nas áreas de manejo florestal, se consideradas as mesmas ocupações e cargas físicas de trabalho (Tabela 5).

Tabela 5. Diferenças médias nos regimes de pausas da NR-15 entre os dois períodos (2015 e 2010), em função das diferenças em IBUTG

\begin{tabular}{ccccc}
\hline \multicolumn{5}{c}{ Pausas segundo a NR-15 (em minutos) } \\
\hline \multicolumn{5}{c}{ Ano } \\
Etapa & 2015 & 2010 & Dif \\
& (min.) & (min.) & (min.) & Perda \\
Corte & 228 & 180 & $+48,0$ & $-8,6$ \\
Pré-arraste & 335 & 307,5 & $+27,5$ & $-3,1$ \\
Arraste & 261 & 177 & $+84,0$ & $-14,6$ \\
\hline
\end{tabular}

Os resultados apresentados, apontaram que houve diferenças nos dimensionamentos dos regimes de pausas. Com base na hipotética aplicação destes regimes, estimou-se os efeitos na produtividade e capacidade de trabalho das ocupações envolvidas na análise. No corte florestal, o regime de pausas estipulado pela NR-15 acarretou em perdas de $8,64 \mathrm{~m}^{3} /$ jornada, durante o $E I$ Niño Godzilla. Na atividade de pré-arraste as perdas foram de 03 toras/jornada de trabalho.

A operação de arraste apresentou queda de produtividade em cerca de 15 toras/jornada. Esta análise foi feita aos moldes da realizada por Kjellstrom et al. (2009a) que estimaram as perdas de produtividade dos trabalhadores em diversos cenários futuros de mudança climática, por meio de temperaturas em IBUTG e regime de pausas estipulado pela norma da NIOSH (National Institute for Occupational Safety and Health). De forma geral, estes resultados demonstraram as diferenças dos regimes de pausas caso a norma NR-15 fosse aplicada na prática.

Por fim, os efeitos negativos do aumento em IBUTG devido ao fenômeno extremo El Niño Godzilla na produtividade dos trabalhadores, foram estimados. Os resultados apontados buscaram demonstrar indícios de alterações no desempenho dos trabalhadores florestais na Amazônia, em decorrência de fenômenos naturais extremos provavelmente ligados à mudança climática.

\section{CONCLUSÃO}

As adequações práticas das condições de exposição ao calor no trabalho, sob o cenário atual e previsto de aumento de temperaturas devem ser consideradas com urgência. A execução efetiva acarretaria em maior bemestar dos trabalhadores, com consequentes melhorias à saúde, à segurança e ao desempenho das operações de exploração de madeira. Esses são requisitos essenciais para a sustentabilidade a longo prazo do manejo florestal na Amazônia.

\section{AGRADECIMENTOS}

Gostaríamos de agradecer à equipe do Laboratório de Manejo Florestal (LMF-INPA) pela geração de ideias para enriquecimento da pesquisa na Amazônia. Por fim, a Mil Madeiras Preciosas Ltda. pela concessão da área para pesquisa e por todo o suporte durante a estadia na empresa.

\section{REFERÊNCIAS}

ALVARES, C.A. et al. Köppen's climate classification map for Brazil. Meteorologische Zeitschrift, v.22, p.1-18, 2013.

BRASIL. Norma Regulamentadora № 15: atividades e operações insalubres. Brasília: Ministério do Trabalho e Emprego, Diário Oficial da União, 1978. Disponível em: http://trabalho.gov.br/index.php/seguranca-e-saudenotrabalho/normatizacao/normas-regulamentadoras Acesso em 30/08/2016.

EMMERT, F. Combinação de dados de campo e métodos computacionais para o planejamento da exploração florestal na Amazônia. 2014. 190 p. (Tese de doutorado)

HÜBLER, M. et al. Costs of climate change: the effects of rising temperatures on health and productivity in Germany. Ecological Economics, v.68, p.381-393. 2008.

IIDA, I. Ergonomia: Projeto e Produção. São Paulo: Edgard Blücher. 1990.

INMET. Banco de dados meteorológicos para ensino e pesquisa. Instituto Nacional de Meteorologia. Disponível em: http://www.inmet.gov.br/portal/index.php?r=bdmep/bdmep

INPE. Instituto Nacional De Pesquisas Espaciais. Disponível em: Http://Clima1.Cptec.Inpe.Br/Estacaochuvosa/Pt. Acesso em $31 / 05 / 2016$.

IPCC. Fourth assessment report: Adaptation and vulnerability. Inter-Governmental Panel on Climate Change. Geneva. Cambridge University Press. 2007.

IPCC. Guidance note for lead authors of the IPCC fifth assessment report on consistent treatment of uncertainties. In: 
IPCC cross-working group meeting on consistent treatment of uncertainties. Inter-Governmental Panel on Climate Change Jasper Ridge, CA, USA. 2010.

JIMÉNEZ-MUÑOZ, J.C. et al. Spatial and temporal patterns of the recent warming of the Amazon Forest. Journal of Geophysical Research: Atmospheres, v.118, p.5204-5215. 2013.

KJELLSTROM, T. Climate change, direct heat exposure, health and well-being in low and middle-income countries. Global Health Action. Guest Editorial. 2009.

KJELLSTROM, T.; CROWE, J. Climate change, workplace heat exposure, and occupational health and productivity in Central America. Climate International Journal of Occupational \& Environmental Health, v.17, n.3, p.270-281, 2011.

KJELLSTROM, T. et al. Workplace heat stress, health and productivity - an increasing challenge for low and middle-income countries during climate change. Global Health Action, v.2, p.16, 2009b.

KJELLSTROM, T. et al. The direct impact of climate change on regional labor productivity. Archives of Environmental \& Occupational Health, v.64, n.4, p.217-227, 2009a.

LEITÃO FILHO, H.F. Considerações sobre a florística de florestas tropicais e subtropicais do Brasil. IPEF, n.35, p.41-46, 1987.

MARENGO, J.A. et al. The drought of 2010 in the context of historical droughts in the Amazon Region. Geophysical Research Letters, v.38, p.1-5, 2011.

MCMICHAEL A.J. et al. Climate change and human health. Geneva: World Health Organization - WHO. 1996.

TRENBERTH, K.E. The definition of El Niño. Bulletin of the American Meteorological Society, v.78, n.12, p.2771-2777, 1997. 